\title{
PAPA and FMF in two siblings: possible amplification of clinical presentation? A case report
}

\author{
Maria Cristina Maggio ${ }^{3^{*}}$, Isabella Ceccherini ${ }^{1}$, Alice Grossi ${ }^{1}$, Marco Gattorno ${ }^{2}$ and Giovanni Corsello ${ }^{3}$
}

\begin{abstract}
Background: Familial Mediterranean Fever is a monogenic autoinflammatory disease, typically characterized by recurrent attacks of fever, serositis, aphthous of oral mucosa, erythema.

"Pyogenic arthritis, pyoderma gangrenosum and acne syndrome" is a rare autoinflammatory disease with variable expression and typically involving joints and skin. Both the diseases are linked by the overproduction of IL-1.

Case presentation: We report on the case of two siblings affected by recurrent attacks of fever, oral aphthous stomatitis, abdominal pain, arthritis, undefined dermatitis at the hands, associated with increased AST, ALT, C-reactive protein, erythrocyte sedimentation rate, serum amyloid A, leucocytosis with neutrophilia. Infectious diseases were excluded. The genetic study for Familial Mediterranean Fever, tumor necrosis factor receptor-associated periodic syndrome, Mevalonate kinase deficiency, showed the homozygous mutation p.M680l of exon 10 in MEFV. Their parents were heterozygous for the same mutation p.M680l, however, the mother showed severe symptoms of FMF (recurrent attacks of fever, arthralgia and arthritis, abdominal pain, thoracic pain), the father showed recurrent pustulosis prevalent on the hands and limbs, with arthralgia and abdominal pain.

Both the patients started colchicine, with an improvement in clinical manifestations and a reduction of serum amyloid A. For the atypical dermatologic signs present in the two siblings and in the father, the study of other autoinflammatory syndromes was performed with next generation sequencing and showed the heterozygous rare missense mutation of unknown significance: p.Val408lle) of PSTPIP1 gene in the two siblings and in the mother, the father was negative. Canakinumab treatment was started in the younger patient, with the resolution of the clinical symptoms and the normalization of serum amyloid $A$.
\end{abstract}

Conclusions: Further studies are needed to better describe the correlation between genotype and phenotype in patients with PAPA syndrome and with PAPA syndrome associated with FMF, considering that the presence of mutations in both genes may amplify clinical presentation and evolution of both diseases.

Keywords: Familial Mediterranean fever, Pyogenic arthritis, pyoderma gangrenosum and acne syndrome, Colchicine, Canakinumab

\footnotetext{
* Correspondence: mariacristina.maggio@unipa.it

${ }^{3}$ Department of Health Promotion Sciences Maternal and Infantile Care, Internal Medicine and Medical Specialities "G. D'Alessandro", University of Palermo, Palermo, Italy

Full list of author information is available at the end of the article
}

(c) The Author(s). 2019 Open Access This article is distributed under the terms of the Creative Commons Attribution 4.0 International License (http://creativecommons.org/licenses/by/4.0/), which permits unrestricted use, distribution, and reproduction in any medium, provided you give appropriate credit to the original author(s) and the source, provide a link to the Creative Commons license, and indicate if changes were made. The Creative Commons Public Domain Dedication waiver (http://creativecommons.org/publicdomain/zero/1.0/) applies to the data made available in this article, unless otherwise stated. 


\section{Background}

Familial Mediterranean Fever (FMF) is a monogenic autoinflammatory disease, secondary to mutations of MEFV gene in the chromosome $16 \mathrm{p} 13$, and typically characterized by recurrent self-limiting attacks of fever, arthritis, aphthous changes in lips and/or oral mucosa, erythema, serositis [1, 2]. FMF is caused by dysregulation of the inflammasome, a complex intracellular multiprotein structure, commanding the overproduction of interleukin-1(IL-1). The attacks of FMF can recognize a trigger in infections, stress, menses.

Pyogenic arthritis, pyoderma gangrenosum and acne (PAPA) syndrome is a rare autosomal dominant autoinflammatory disease with incomplete penetrance and variable expression. It involves the proline serine threonine phosphatase-interacting protein 1 (PSTPIP1/CD2BP1) gene on chromosome $15 \mathrm{q}$.

The mutations first described, A230T and E250A, as well as other mutations found on the same gene, increase phosphorylation of PSTPIP1, inducing a higher affinity for the linkage with pyrin than wild type forms [3]. Hence, inflammasome assembly and activation is altered, increasing release of IL-1 beta [4]. The overexpression of IL-1 beta induces an increased secretion of proinflammatory chemokines and cytokines, with the recruitment and activation of neutrophils, managing a neutrophil-mediated inflammatory disease.

This mutation causes the overproduction of the proinflammatory cytokine IL-1.

This discovery linked FMF and PAPA syndrome within the same previously described pathway, suggesting a common pathway that could be targeted therapeutically.

The clinical phenotype of PAPA syndrome is characterized by early onset of recurrent episodes of acute aseptic inflammation of the joints, generally occurring in the first two decades of life, followed by typical skin lesions in the third decade, after a clear reduction of the arthritis.

\section{Case presentation}

We report on two siblings, affected by recurrent attacks of fever, oral aphthous stomatitis, abdominal pain, retrosternal and thoracic pain, arthritis, undefined dermatitis at the hands, with palmar maculopapular erythema followed by desquamation. Increased AST, ALT (1.5 x n.v.), C-reactive protein (CRP): $20 \mathrm{x}$ n.v.; erythrocyte sedimentation rate (ESR): > 100, leucocytosis with neutrophilia were present in both patients. Specific IgM and IgG to exclude infection of Epstein-Barr virus [5], Parvovirus, HHV6, Mycoplasma Pneumoniae, pharyngeal swab for Streptococcus pyogenes were negative.

Patient 1 is a 16-year-old boy, with recurrent attacks, 37 days lasting, of fever, oral aphthous stomatitis, abdominal pain, thoracic pain, arthritis, lumbar pain, palmar maculopapular erythema followed by desquamation, periungual dermatitis with peeling, erythema, acne.
Patient 2 is an 8.4-year-old boy, with recurrent, 3-7 days lasting, attacks of fever, oral aphthous stomatitis, abdominal pain, diarrhoea, vomiting, with -3 episodes/year of acute abdomen, mimicking acute appendicitis and requiring recovering in surgery emergency unit, retrosternal and thoracic pain, arthritis, dermatitis at the hands, rash at the trunk and at the face, with palmar maculopapular erythema followed by desquamation.

The mother showed recurrent episodes of fever with arthralgia and arthritis, headache, asthenia, abdominal pain, thoracic pain; the father showed recurrent pustulosis prevalent on the hands and limbs, with arthralgia and abdominal pain.

The genetic study for FMF, TNF receptor-associated periodic syndrome (TRAPS), Mevalonate kinase deficiency (MVK) showed the same genetic profile in the two patients. They showed the homozygous mutation p.M680I of exon 10.

Both parents and the 18-year-old sister showed a heterozygous mutation of p.M680I.

The two patients showed increased levels of serum amyloid A (SAA) (> 5-10 x n.v.) far away from recurrent attacks.

Patient 2 started colchicine, with a reduction of the number and length of fever episodes, abdominal pain, arthritis, aphthous stomatitis. However, abdominal pain, arthralgia, vomiting, diarrhoea, dermatitis persisted. SAA levels reduced, still continuing to maintain elevated levels in the free intervals.

For the atypical dermatologic signs present in the two siblings and in the father, the study of other autoinflammatory syndromes was performed using a specific next generation sequencing based gene panel already reported [6]. This allowed to detect a heterozygous rare missense mutation of unknown significance in the two patients and in the mother, the p.(Val408Ile) in the exon 15 of the PSTPIP1 gene.

Patient 1 is continuing colchicine at the dosage of 1 , $25 \mathrm{mg} /$ day, with the resolution of recurrent attacks of fever, serositis, aphthous stomatitis. However, maculopapular erythema followed by desquamation, periungual dermatitis with peeling and acne persist and he is in follow-up to consider the treatment with canakinumab, a fully human anti-IL-1beta monoclonal antibody will be started in the next months.

Patient 2 started subcutaneous treatment with canakinumab, at the dosage of $2 \mathrm{mg} / \mathrm{kg}$ every 4 weeks, maintaining colchicine. After the first 3 doses, fever, abdominal pain, arthralgia persisted even if less severe for intensity and length. The canakinumab dosage was increased at $4 \mathrm{mg} / \mathrm{kg} /$ every 4 weeks and the symptoms resolved, with the complete normalization of SAA. However, for the persistence of diarrhoea, colchicine dose will be reduced progressively. 


\section{Discussion and conclusions}

The homozygous mutation M680I of MEFV is typically associated with a severe FMF phenotype. The same mutation has been observed also in a remarkable number of heterozygous patients affected with FMF symptoms and FMF-like pictures [7]. This implies that the clinical picture of probands'parents, both carriers of one M680I allele, can be reconducted to their MEFV genotype.

The detection of the rare missense mutation p.(Val408Ile) of the PSTPIP1 gene suggests some considerations. The rarity of the mutation, with an in-silico prediction to be damaging though it is still classified as a variant of unknown significance (VUS), in association with a more severe presentation of FMF, has prompted us to take this variant into consideration as a modifier allele.

In our patients, skin lesions were significantly expressed and did not respond to colchicine, however, they were not typical of PAPA Syndrome. In patient 2, canakinumab satisfactory controlled the clinical presentation, with the normalization of SAA. The father showed skin lesions not specific of the PSTPIP1 gene mutation, and he is consistently negative for the presence of any possibly PSTPIP1 causative variant. Other contributory genetic elements able to explain his dermatological manifestations could be investigated in the future by exome study.

In our patients, the association of MEFV and PSTPIP1 mutations, rarely described in the same subject, could represent the amplifying factor of a meaningful clinical picture, expressed as FMF refractory to colchicine treatment, with a more severe clinical presentation, as observed in the two siblings (homozygous however for a significantly severe mutation of exon 10) and in the mother. As the father, she is heterozygous for the same mutation, however, she presents clinical features much more severe, with a poor quality of life.

Significant variation is described in PAPA syndrome presentation from case to case; it is conceivable that the spectrum of the disease is wider than currently seen. Furthermore, the association in the same patient of mutations in the MEFV gene, involving the inflammasome as well, may increase the clinical expression of the disease thus requiring treatment with high dose of anti-IL-1 drugs. Efficacy and safety profile also in paediatric age [8-10] permit to consider canakinumab in low responders to colchicine. Further studies are needed to better describe the correlation between genotype and phenotype in patients with PAPA syndrome and with PAPA syndrome associated with FMF, considering that the presence of mutations in both genes may amplify clinical presentation and evolution of both diseases.

\section{Abbreviations}

FMF: Familial Mediterranean Fever; CRP: C-reactive protein; ESR: Erythrocyte sedimentation rate; IL-1: Interleukin-1; MVK: Mevalonate kinase deficiency; PAPA syndrome: Pyogenic arthritis, pyoderma gangrenosum and acne;
PSTPIP1: Proline serine threonine phosphatase-interacting protein 1; SAA: Serum amyloid A; TRAPS: TNF receptor-associated periodic syndrome; VUS: Variant of unknown significance

\section{Acknowledgements}

not applicable.

\section{Authors' contributions}

Maria Cristina Maggio: prepared the case presentation, followed the patient, and wrote the paper; Isabella Ceccherini and Alice Grossi: performed the genetic study of the patients; Isabella Ceccherini, Marco Gattorno and Giovanni Corsello: revised the paper. All authors read and approved the manuscript.

Funding

no funding is declared.

Availability of data and materials

materials and data of the patient are included in the medical records of the patient.

Ethics approval and consent to participate not applicable.

\section{Consent for publication}

Consent for publication was obtained from the parents of the child. The consent is included in the medical records of the patient.

Competing interests

The authors report no conflicts of interest in this work.

\section{Author details}

${ }^{1}$ Genetic Unit, IRCCS Istituto Giannina Gaslini, Genoa, Italy. ${ }^{2}$ Clinica Pediatrica e Reumatologia, Istituto Giannina Gaslini, Genoa, Italy. ${ }^{3}$ Department of Health Promotion Sciences Maternal and Infantile Care, Internal Medicine and Medical Specialities "G. D’Alessandro", University of Palermo, Palermo, Italy.

Received: 18 July 2019 Accepted: 9 August 2019

Published online: 23 August 2019

\section{References}

1. Gattorno M, Hofer M, Federici S, Vanoni F, Bovis F, Aksentijevich I, et al. Eurofever Registry and the Paediatric Rheumatology International Trials Organisation (PRINTO). Classification criteria for autoinflammatory recurrent fevers. Ann Rheum Dis. 2019. pii: annrheumdis-2019-215048. https://doi. org/10.1136/annrheumdis-2019-215048.

2. Papa R, Doglio M, Lachmann HJ, Ozen S, Frenkel J, Simon A, et al. Paediatric Rheumatology International Trials Organisation (PRINTO) and the Eurofever Project. A web-based collection of genotype-phenotype associations in hereditary recurrent fevers from the Eurofever registry. Orphanet J Rare Dis. 2017;12(1):167. https://doi.org/10.1186/s13023-017-0720-3.

3. Shoham NG, Centola M, Mansfield E, Hull KM, Wood G, Wise CA, et al. Pyrin binds the PSTPIP1/CD2BP1 protein, defining familial Mediterranean fever and PAPA syndrome as disorders in the same pathway. Proc Natl Acad Sci U S A. 2003;100:13501-6.

4. Marzano AV, Damiani G, Genovese, G, Gattorno M. A dermatologic perspective on autoinflammatory diseases. Clin Exp Rheumatol. 2018 JanFeb;36 Suppl 110(1):32-38. Epub 2018.

5. Maggio MC, Liotta A, Cardella F, Corsello G. Stevens-Johnson syndrome and cholestatic hepatitis induced by acute Epstein-Barr virus infection. Eur J Gastroenterol Hepatol. 2011;23(3):289.

6. Rusmini M, Federici S, Caroli F, Grossi A, Baldi M, Obici L, et al. Nextgeneration sequencing and its initial applications for molecular diagnosis of systemic auto-inflammatory diseases. Ann Rheum Dis. 2016; 75(8):1550-7.

7. Cekin N, Akyurek ME, Pinarbasi E, Ozen F. MEFV mutations and their relation to major clinical symptoms of familial Mediterranean fever. Gene. 2017;626:9-13.

8. De Benedetti F, Gattorno M, Anton J, Ben-Chetrit E, Frenkel J, Hoffman HM, et al. Canakinumab for the Treatment of Autoinflammatory Recurrent Fever Syndromes. N Engl J Med. 2018;378(20):1908-19. 
9. Sota J, Vitale A, Insalaco A, Sfriso P, Lopalco G, Emmi G, et al. "Working Group" of Systemic Autoinflammatory Diseases of SIR (Italian Society of Rheumatology). Safety profile of the interleukin-1 inhibitors anakinra and canakinumab in real-life clinical practice: a nationwide multicenter retrospective observational study. Clin Rheumatol. 2018;37(8):2233-40.

10. Omenetti A, Carta S, Delfino L, Martini A, Gattorno M, Rubartelli A. Increased NLRP3-dependent interleukin $1 \beta$ secretion in patients with familial

Mediterranean fever: correlation with MEFV genotype. Ann Rheum Dis. 2014 Feb;73(2):462-9.

\section{Publisher's Note}

Springer Nature remains neutral with regard to jurisdictional claims in published maps and institutional affiliations.

Ready to submit your research? Choose BMC and benefit from:

- fast, convenient online submission

- thorough peer review by experienced researchers in your field

- rapid publication on acceptance

- support for research data, including large and complex data types

- gold Open Access which fosters wider collaboration and increased citations

- maximum visibility for your research: over $100 \mathrm{M}$ website views per year

At $\mathrm{BMC}$, research is always in progress.

Learn more biomedcentral.com/submissions 